Molly Hoss, MD;

Corey Lyon, DO

University of Colorado

Family Medicine Residency,

Denver

DEPUTY EDITOR

Shailendra Prasad, MBBS,

MPH

University of Minnesota

North Memorial Family

Medicine Residency

Program, Minneapolis

\title{
Do-it-yourself cervical cancer screening?
}

\author{
Enlisting patients to self-collect specimens for human \\ papillomavirus testing is an effective way to detect \\ cervical precancer in those who might not otherwise \\ undergo cervical cancer screening.
}

\section{PRACTICE CHANGER}

Have patients who decline a pelvic examination self-collect a specimen for human papillomavirus polymerase chain reaction testing as an alternative to a clinician-collected one.

STRENGTH OF RECOMMENDATION

B: Meta-analysis of observational trials. ${ }^{1}$

Arbyn M, Smith SB, Temin S, et al; Collaboration on Self-Sampling and HPV Testing. Detecting cervical precancer and reaching underscreened women by using HPV testing on self-samples: updated metaanalyses. $B M J$. 2018;363:k4823.

\section{ILLUSTRATIVE CASE}

A 40-year-old woman presents to your office to establish care. During your interview you realize that she has never been screened for cervical cancer. In fact, she has not had a pelvic exam because she is fearful of the procedure. She would like to know if alternatives exist for cervical cancer screening. What can you suggest?

A lthough deaths from cervical cancer decreased in the United States from 1975 to 2017, demographic and social disparities in the burden of the disease remain. ${ }^{2,3}$ Data from 2016 reveal that cervical cancer incidence per 100,000 women is lowest among white (7.5), Asian-Pacific Islander (5.8), and American Indian/Alaska native (5.6) women, and highest among Hispanic (9.8) and black (8.7) women, which could be explained by lower screening rates in these populations. $^{4,5}$ The National Cancer Institute's publication on reducing cancer health disparities states that the most effective way to reduce cervical cancer incidence and mortality is by increasing screening rates among women who have not been screened or who have not been screened regularly. ${ }^{6}$

The US Food and Drug Administration (FDA) approved the first human papillomavirus (HPV) screening test in $2003 .{ }^{7}$ Evidence now suggests that high-risk HPV screening provides greater protection against cervical cancer than screening with cytology alone. ${ }^{8}$ The American College of Obstetricians and Gynecologists (ACOG) and the US Preventive Services Task Force (USPSTF) have changed their recommendations to include primary HPV testing as an alternative method to Pap smears for cervical cancer screening. ${ }^{9}$

An advantage of primary HPV screening is that it can be performed on a specimen collected by the patient, which could potentially increase rates of screening and help to decrease demographic and social disparities. A randomized trial of almost 2000 women ages 21 to 65 years that evaluated the acceptability of this method to patients revealed that more than half of women prefer the idea of a self-collected specimen to one that is collected by a clinician because it is more convenient and obviates the need for a pelvic exam. ${ }^{10}$

A meta-analysis of 36 studies and more than 150,000 women concluded that when 
self-collected samples were used with signalbased assays, the tests were not as sensitive or specific as when clinician-collected samples were used. ${ }^{11}$ However, the meta-analysis also found that some polymerase chain reaction (PCR)-based HPV tests were similarly sensitive for both self- and clinician-collected samples.

\section{STUDY SUMMARY}

\section{PCR vs signal amplification HPV tests with collection by patients vs clinicians}

This meta-analysis compared the accuracy of high-risk HPV self-screening with clinician collection of samples (56 diagnostic accuracy trials; total $\mathrm{N}$ not provided) in identifying cervical intraepithelial neoplasia grade 2 or worse (CIN 2+) with signal amplification and PCR tests evaluated separately. ${ }^{1}$ In addition, this review evaluated strategies to screen women who are underscreened or not screened, which was defined as women who were irregularly or never screened, or did not respond to reminder letters about cervical cancer screening (25 randomized controlled trials [RCTs]; total N not provided).

In the diagnostic accuracy studies, patients collected a vaginal sample themselves and then had a sample taken by a clinician. CIN 2+ or 3+ was confirmed by either colposcopy and biopsy performed on all patients or by a positive high-risk HPV test result. Studies were further divided into those using assays based on signal amplification or PCR.

In signal amplification assays, the pooled sensitivity for CIN 2+ was lower in the group with the self-collected samples than in the clinician-collected sample group (77\%; $95 \%$ confidence interval [CI], $69 \%-82 \%$ vs $93 \%$; 95\% CI, 89\%-96\%). The pooled specificity to exclude CIN 2+ was also lower in the group with the self-collected samples (84\%; 95\% CI, $77 \%-88 \%$ vs $86 \%$; $95 \%$ CI, $81 \%-90 \%)$. In highrisk HPV assays based on PCR, there was no difference in sensitivity (96\%) or specificity (79\%) between the specimen groups.

With regard to the pooled relative sensitivity and specificity of signal amplification assays, those using self-swab samples were less sensitive and less specific for CIN $2+($ sensitivity ratio $=0.85 ; 95 \% \mathrm{CI}, 0.80-0.89$; specificity ratio $=0.96 ; 95 \% \mathrm{CI}, 0.93-0.98$ ) and CIN 3+ (sensitivity ratio $=0.86 ; 95 \% \mathrm{CI}$, $0.76-0.98$; specificity ratio $=0.97 ; 95 \% \mathrm{CI}$, 0.95-0.99). Using PCR assays, there was no difference between groups in relative sensitivity for the diagnosis of CIN 2+ (sensitivity ratio $=0.99 ; 95 \% \mathrm{CI}, 0.97-1.02$ ) and CIN 3+ (sensitivity ratio $=0.99 ; 95 \% \mathrm{CI}, 0.96-1.02$ ). Relative specificity was slightly lower in the self-swab group for CIN 2+ (specificity ratio = 0.98; 95\% CI, 0.97-0.99) and CIN 3+ (specificity ratio $=0.98 ; 95 \% \mathrm{CI}, 0.97-0.99$ ).

- The second analysis to evaluate which outreach strategies are effective methods for screening underscreened/unscreened women found that delivering self-sample kits to patients was more effective than the control method, which was sending reminders to women to undergo conventional screening (95\% vs 53\%; mean difference [MD], 41\%; 95\% CI, 3\%-78\%). Similarly, mailing kits to patients compared favorably to the control method (25\% vs $12 \%$; MD, $13 \%$; $95 \%$ CI, $10 \%$ $15 \%)$.

\section{WHAT'S NEW}

\section{Self-collected specimens can be as reliable as clinician-collected ones}

This is the first study to provide robust evidence that high-risk HPV PCR-based assays using patient self-collected specimens are as sensitive at diagnosing CIN 2+ or 3+ as using clinician-collected samples.

\section{CAVEATS}

\section{Balancing lower specificity with reaching underscreened populations}

Patients with a positive HPV test result require additional testing. The success rates for this follow-up are not known and could be a barrier to accurate diagnoses because of accessibility and patient willingness to follow up with a pelvic exam. In addition, selfcollection may be less specific than cytology and could increase colposcopy referrals that lead to negative findings and overtreatment. ${ }^{12}$ However, the increased acceptance of this screening method could make it an effective strategy to reach underscreened or reluctant patients.
This study offers robust evidence that high-risk HPV PCR-based assays using patient-collected specimens are as sensitive at diagnosing CIN 2+ or 3+ as using cliniciancollected samples. 


\section{CHALLENGES TO IMPLEMENTATION}

Availability of PCR-based HPV assays may be an issue

HPV PCR assays may not be available at all laboratories, but signal amplification HPV tests have been shown to be inferior to PCR assays. Physicians will have to confirm with their laboratories whether PCR-based HPV assays are available.

\section{ACKNOWLEDGEMENT}

The PURLs Surveillance System was supported in part by Grant Number UL1RR024999 from the National Center for Research Resources, a Clinical Translational Science Award to the University of Chicago. The content is solely the responsibility of the authors and does not necessarily represent the official views of the National Center for Research Resources or the National Institutes of Health. Copyright @ 2020. The Family Physicians Inquiries Network. All rights reserved.

\section{References}

1. Arbyn M, Smith SB, Temin S, et al; Collaboration on Self-Sampling and HPV Testing. Detecting cervical precancer and reaching underscreened women by using HPV testing on self-samples: updated meta-analyses. BMJ. 2018;363:k4823.

2. National Cancer Institute Surveillance, Epidemiology, and End Results Program. Cancer stat facts: cervical cancer. www.seer. cancer.gov/statfacts/html/cervix.html. Accessed June 29, 2020.

3. Singh GK, Azuine RE, Siahpush M. Global inequalities in cervical cancer incidence and mortality are linked to deprivation, low socioeconomic status, and human development. Int J MCH AIDS 2012;1:17-30.

4. US Cancer Statistics Working Group. US Cancer Statistics Data Visualizations Tool, based on November 2018 submission data (1999-2016): US Department of Health and Human Services, Centers for Disease Control and Prevention and National Cancer Institute. June 2019. www.cdc.gov/cancer/dataviz. Accessed June 29, 2020.

5. MacLaughlin KL, Jacobson RM, Breitkopf CR, et al. Trends over time in Pap and Pap-HPV cotesting for cervical cancer screening. J Womens Health. 2019;28:244-249.

6. Freeman HP, Wingrove BK. Excess Cervical Cancer Mortality: A Marker for Low Access to Health Care in Poor Communities. NIH Pub. No. 05-5282. Rockville, MD: National Cancer Institute, Center to Reduce Cancer Health Disparities, May 2005. www. cancer.gov/about-nci/organization/crchd/about-healthdisparities/resources/excess-cervical-cancer-mortality.pdf. Accessed June 29, 2020.

7. FDA approves expanded use of HPV test. Infection Control Today March 31, 2003. www.infectioncontroltoday.com/guidelines/ fda-approves-expanded-use-hpv-test. Accessed June 29, 2020.

8. Ronco G, Dillner J, Elfström K, et al. Efficacy of HPV-based screening for prevention of invasive cervical cancer: follow-up of four European randomised controlled trials. Lancet. 2014;383: 524-532.

9. CDC. Cervical cancer screening guidelines for average-risk women. www.cdc.gov/cancer/cervical/pdf/guidelines.pdf. Accessed June 29, 2020.

10. Mao C, Kulasingam S, Whitham $\mathrm{H}$, et al. Clinician and patient acceptability of self-collected human papillomavirus testing for cervical cancer screening. J Womens Health. 2017;26:609-615.

11. Arbyn M, Verdoodt F, Snijders PI, et al. Accuracy of human papillomavirus testing on self-collected versus clinician-collected samples: a meta-analysis. Lancet Oncol. 2014;15:172-183.

12. Lazcano-Ponce E, Lorincz A, Cruz-Valdez A, et al. Self-collection of vaginal specimens for human papillomavirus testing in cervical cancer prevention (MARCH): a community-based randomised controlled trial. Lancet. 2011;378:1868-1873.
CONTINUED FROM PAGE 271

found on our Web site at www.mdedge.com/ familymedicine. We remain committed to supplying you with all of the information you need to provide your patients with the very best care - no matter what brings them in to see you.

JFP
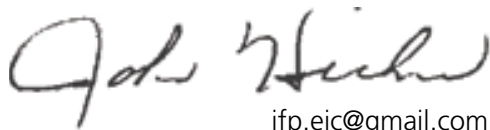

jfp.eic@gmail.com
References

1. Low-cost dexamethasone reduces death by up to one third in hospitalized patients with severe respiratory complications of COVID-19. Recovery: Randomised Evaluation of COVID-19 Therapy Web site June 16, 2020. www recoverytrial.net/ news/low-cost-dexamethasone-reduces-death-by-up-toone-third-in-hospitalised-patients-with-severe-respiratorycomplications-of-covid-19. Accessed July 1, 2020.

2. Beigel JH, Tomashek KM, Dodd LE, et al. Remdesivir for the treatment of Covid-19-preliminary report [published online ahead of print]. N Engl J Med. doi: 10.1056/NEJMoa2007764.
3. Li L, Zhang W, Hu Y, et. al. Effect of convalescent plasma therapy on time to clinical improvement in patients with severe and lifethreatening COVID-19: a randomized clinical trial [published online ahead of print]. JAMA. doi:10.1001/jama.2020. 10044.

4. Stokes EK, Zambrano LD, Anderson KN, et al. Coronavirus disease 2019 case surveillance-United States, January 22-May 30, 2020. MMWR Morb Mortal Wkly Rep. 2020;69:759-765.

5. Xu J, Murphy SL, Kochanek KD, et al. Mortality in the United States, 2018. NCHS Data Brief. 2020;1-8. 